bach, M. (Hg.): Sicherheitskonzepte für das Internet. Berlin, S. 7-26; http://www.inf.ethz.ch/vs/publ/ papers/allgegenwaertig.pdf (download 5.5.08)

Nentwich, M.; Peissl, W. (Hg.), 2005: Technikfolgenabschätzung in der österreichischen Praxis. Festschrift für Gunther Tichy, Wien

OECD - Organisation for Economic Co-operation and Development, 1980: Guidelines on the Protection of Privacy and Transborder Flows of Personal Data; http://www.oecd.org/document/18/0,3343,en_2649_3 4255_1815186_1_1_1_1,00.html (download 5.5.08)

OECD - Organisation for Economic Co-operation and Development, 2003: Kurzfassung OECDRichtlinien über Datenschutz und grenzüberschreitende Ströme personenbezogener Daten; http://www.oecd.org/dataoecd/16/7/15589558.pdf (download 5.5.08)

Varshney, U., 2003: Pervasive healthcare. In: IEEE Computer 36/12 (2003), S. 138-140

Weiser, M., 1991: The computer for the $21^{\text {st }}$ century. In: Sci Amer 265/3 (1991), S. 66-75; http://www.ubiq.com/hypertext/weiser/SciAmDraft3. html (download 5.5.08)

Weiser, M.; Brown, J.S., 1996: The Coming Age of Calm Technology; http:/www.ubiq.com/hypertext/ weiser/acmfuture2endnote.htm (download 5.5.08)

\author{
Kontakt \\ Johann Čas \\ Institut für Technikfolgen-Abschätzung \\ Österreichische Akademie der Wissenschaften \\ Strohgasse 45/3. Stock, 1030 Wien, Österreich \\ E-Mail: jcas@oeaw.ac.at \\ Internet: http://www.oeaw.ac.at/ita/
}

\section{Ethische Fragen des Pervasive Computing im Gesundheitswesen}

\author{
von Ludwig Siep, Universität Münster
}

Geräte und Systeme des Pervasive Computing versprechen im Gesundheitswesen eine Reihe von Vorteilen für die Benutzer und die Gesellschaft. Für Krankenhäuser und andere medizinische Einrichtungen (z. B. die Unfallmedizin) stellen sie Verbesserungen in der Kommunikation und der Effizienz, aber auch Einsparmöglichkeiten in Aussicht. Für Kranke oder in ihren körperlichen und mentalen Funktionen Eingeschränkte bieten sie Überwachungs- und Kompensationsmöglichkeiten, die das alltägliche Leben erleichtern und ihre Selbstständigkeit bzw. Unabhängigkeit von Pflegepersonal und -einrichtungen steigern können. Neben diesen positiven Effekten sind aber auch gravierende Risiken für Sicherheit und Autonomie der Patienten sowie für die Gerechtigkeit im Gesundheitswesen erkennbar. Die ethische Reflexion sollte frühzeitig auf diese Probleme aufmerksam machen, ohne von den Entwicklungsmöglichkeiten abraten zu müssen.

Unter Pervasive Computing im Gesundheitswesen versteht man den umfassenden Einsatz von mobilen, drahtlosen, oft miniaturisierten Geräten der Datenerfassung, -verarbeitung und -übertragung im Bereich der Prävention, Diagnostik, Therapie und Krankenpflege. Auch Anwendungen im Bereich der Altenpflege oder die Verwendung technischer Geräte zum Ausgleich eingeschränkter Fähigkeiten (Hör-, Sehoder Gehhilfen, Kompensation kognitiver Defizienzen etc.) gehören dazu. „Pervasive“ bedeutet auch, dass die Geräte möglichst unauffällig und ,benutzerfreundlich“ in der Umgebung des Trägers - vom Körper über die Kleidung bis in die Wohnung - integriert sind (,They are everywhere“, Korhonen, Bardram 2004, S. 229). Zu ihrem spezifischen Charakter gehört in der Regel ihre „Intelligenz“, d. h. ihre Selbstlokalisation und die Erfassung ihrer Umgebung (,context awareness“) sowie ihre (kybernetische) Fähigkeit, auf die erfassten Daten (vor allem dramatische Änderungen) durch 
entsprechende „outputs“ (Signale oder Impulse) zu reagieren. Diese Reaktion kann aufgrund einer Rückkopplung mit anderen Geräten oder Stationen erfolgen. Insofern es solche Geräte oft mit der Übertragung von Daten an räumlich entfernte Stationen (Arztpraxis, Krankenhaus etc.) zu tun haben, überschneiden sich die technischen und ethischen Fragen mit denen der sogenannten „Telemedizin“ (Siep 2007).

Ethik hat mit der Bewertung von Zielen des menschlichen Handelns zu tun. Dabei spielen die Ansprüche der Menschen eine besondere Rolle, sowohl die ,negativer Art", auf Vermeidung von Zwang und Schaden orientierten, wie die positiven, nämlich der Anspruch auf Kooperation, Hilfe und Unterstützung, die auch über strikte Rechtsansprüche hinausgehen. Diese Güter und Rechte unterliegen einer historischen Entwicklung, die in verschiedenen Kulturen unterschiedlich verlaufen kann. In der modernen europäischen Kulturentwicklung hat etwa das Gut der individuellen Autonomie zunehmend an Gewicht gewonnen. In der Medizin zeigt sich das im Übergang von einem paternalistischen, überwiegend vom Expertenurteil bestimmten Verhältnis zum Patienten zu einem partnerschaftlichen, das von der informierten Zustimmung des Kranken oder sogar vom Wunsch nach „Dienstleistung“" geprägt ist. Mit der erheblichen Ausweitung der Handlungsmöglichkeiten der Medizin und ihren Erfolgen in der Lebensverlängerung und -verbesserung ist aber auch die Nachfrage nach medizinischen Leistungen gestiegen. Das hat u. a. zu einer Verknappung der Ressourcen geführt. Damit gewinnt in den neueren medizinethischen und gesundheitspolitischen Debatten auch das Gut der Verteilungsgerechtigkeit eine immer größere Bedeutung.

Dass die Möglichkeiten des Pervasive Computing das Wohlergehen der Benutzer erhöhen können, scheint unbestreitbar. Gewinnen kann vor allem ihre Sicherheit vor Gefahren und unbemerkten Schädigungen, aber auch ihre unbehinderte selbstbestimmte Lebensführung. Zugleich bringt die Anwendung dieser Geräte und Systeme aber auch Gefahren mit sich, vor allem für die Autonomie, aber auch für das Wohlergehen und die Gerechtigkeit. Darauf soll im Folgenden unter den Stichworten "Sicherheit“, „Autonomie“ und „Gerechtigkeit" eingegangen werden.

\section{Erhöhung oder Gefährdung der Sicherheit?}

Dass ein besserer Zugang und Austausch der medizinischen Daten und ihre Fernübermittlung an medizinische Einrichtungen die Sicherheit von Patienten erhöhen kann, ist seit längerem ein Argument für die Entwicklung von Systemen des Pervasive Computing und der Telemedizin. Es ist bekannt, wie viele Fehlbehandlungen auf mangelnde Information über die Krankengeschichte und die Behandlung von Patienten zurückgehen. Wichtig ist sicher auch der Zeitgewinn durch Fernübertragung von Daten etwa im Bereich der Unfallmedizin. Die Verkleinerung und Tragbarkeit der Geräte bringt daher zweifellos Vorteile. Das gilt auch für die Überwachungs- und Behandlungsgeräte im Krankenhaus und für die Kommunikation der in großen Krankenhäusern zwischen verschiedenen Stationen und Labors geradezu „nomadisierenden“ Ärzte, Pfleger, manchmal auch Patienten (Bardram et al. 2007, S. 55). Erhebliche Vorteile versprechen auch die vor allem von Risikopatienten - sei es als externes Gerät z. B. integriert in die Kleidung oder als Implantat - zu tragenden diagnostischen oder therapeutischen Systeme. Diese Vorzüge erhöhen sich in dem Maße, in dem die Verfügbarkeit medizinischer Dienste, aber auch stationärer oder mobiler Pflegeeinrichtungen abnimmt - ein erwartbares Resultat der Alterungsprozesse in der Bevölkerung.

In zwei Hinsichten enthalten diese Prozesse gleichwohl auch Gefährdungspotenziale, auf die der Ethiker aufmerksam machen muss: Zum einen durch mögliche Defekte oder Schwierigkeiten in der Bedienung (a), zum anderen durch ein Übermaß an präventiven Einstellungen, die den Wert der gegenwartsbezogenen Gesundheit und Lebensfreude beeinträchtigen (b).

Ad (a): Komplizierte technische Geräte sind störungsanfällig. Sie müssen oft sorgfältig gewartet werden (analoge Beispiele sind die Luftfahrt oder die Kernenergie) und ihre Bedienung stellt oft erhebliche Anforderungen vor allem dann, wenn ständig erneuerte und verbesserte Versionen auf dem Markt angeboten werden. Bedienungs- und Wartungsfehler oder Defekte von Mikroimplantaten oder Sensoren in der Kleidung können erhebliche Risiken darstellen, besonders wenn sich der Träger und die „entlasteten“ Akteure des Gesund- 
heitswesens auf diese Geräte verlassen. Bis zu einem gewissen Grade mögen immerhin die Vernetzungen zu einer wechselseitigen Kontrolle der Systeme führen.

Es ist daher auch ein Hauptziel der Entwicklung von Pervasive Computing, bedienungsfreundliche und an die Fähigkeiten der Benutzer angepasste Systeme zu konstruieren (Lukowicz et al. 2004, S. 232; Korhonen, Bardram 2004, S. 229). Auch Fehlertoleranz sollte ein Entwicklungsziel sein. Gerade im Bereich der Altenpflege muss verhindert werden, dass der Benutzer solcher Systeme bei Defekten mit möglicherweise gravierenden Folgen in eine Situation der Hilflosigkeit gerät, wie viele im Alltag etwa bei Computerproblemen oder vor Fahrkartenautomaten in fremden Städten. Die Diskrepanz zwischen dem, was sich den ,mit dem Computer Aufgewachsenen" und den Mitgliedern der älteren Generation als Probleme darstellen, wird vermutlich noch für einige Zeit erhalten bleiben.

Ad (b): Der zweite Aspekt der Probleme im Bereich der Sicherheit besteht in der $\mathrm{Zu}-$ nahme des präventiven Denkens im gesamten Bereich der Lebensführung. Frühdiagnostik und Prävention haben in der modernen Medizin ohnehin einen enormen Aufschwung genommen - oft keineswegs ausgeglichen durch einen entsprechenden Zuwachs an therapeutischen Möglichkeiten (man denke an die Gendiagnostik). Die dadurch entstehende Belastung durch die Erwartung ungewisser, aber oft kaum vermeidbarer Erkrankungen ist bekannt. Durch die Einführung diagnostischer Geräte in den alltäglichen Lebensbereichen (,wearable technology") könnte sich diese Tendenz verstärken. Nicht nur besonders Risikogefährdete, sondern auch „Normalverbraucher“ könnten, vor allem bei steigendem Lebensalter, immer mehr Aufmerksamkeit auf das Erfassen von Daten und das Beobachten ihres Körpers richten. ${ }^{1}$ Am Ende würde man sich zu seinem Körper verhalten wie ein Pilot im Cockpit oder ein Lotse im Tower. Die Folge kann - wie schon heute häufig etwa beim Benutzen von Blutdruckmessgeräten - eine ständige, oft bis ins Depressive gesteigerte Sorge vor Gefährdungen sein. Es kann sogar insgesamt zu einer Art Erosion von „Gesundheit" kommen. Denn diese besteht ja auch im Vergessen oder Übersehen alltäglicher Risiken und Beeinträchtigungen. Es ist eine der wohltätigsten Wirkungen der menschlichen Gewohnheit, dass sie Störungen und Schmerzen (bis zu einem gewissen Grad) sozusagen unter die Aufmerksamkeitsschwelle drückt und so erträglich macht - vor allem dann, wenn die Aufmerksamkeit durch andere, neue oder „wichtigere“ Dinge abgelenkt wird. Im Übrigen kennen wir die Gefahren eines übersteigerten Sicherheits- und Präventionsbedürfnisses auch aus den sozialen Konstellationen der „inneren" und ,äußeren" Sicherheit.

Weiterhin ist $u$. a. aus der GendiagnostikDebatte bekannt, dass solche Einstellungen individuell stark variieren. Was bei dem einen ein Gefühl der Sicherheit auslösen mag, kann bei dem anderen zu erheblichen Belastungen führen. Ethisch wichtig ist daher, dass kein Druck oder sozialer Zwang zur Benutzung überwachender oder „frühwarnender“ Geräte ausgeübt wird. Der Markt an „Funktionskleidung“ mit Messgeräten für körperliche und Umweltdaten darf nicht dazu führen, dass Menschen ohne besondere Risiken nur noch mit der Selbstbeobachtung und der Vorsorge für mögliche Beeinträchtigungen beschäftigt sind. Zumal dann, wenn Prävention als ein Mittel der Sparsamkeit gefördert wird - etwa auch durch die Raten bzw. Prämien von Krankenversicherungen.

\section{Stärkung der Autonomie oder „Großer Bruder"?}

Es ist kaum zu bestreiten, dass unauffällige Hör-, Gleichgewichts- oder Gedächtnishilfen (etwa Erinnerungen an die Einnahme wichtiger Medikamente) ein selbstbestimmtes Leben unterstützen bzw. bewahren können (z. B. im Alter). Das gilt vor allem bei abnehmender Verfügbarkeit menschlicher Hilfe, etwa in Notfällen oder im Alter, verstärkt durch die Knappheit an Pflegepersonal und -einrichtungen. Die Anstrengungen der Technologieentwickler, etwa computerisierte Kleidung so unauffällig, störungsfrei und automatisiert herzustellen, dass sie den alltäglichen Lebensablauf und die sozialen Beziehungen nicht beeinträchtigen, gehen daher sicher in die richtige Richtung: „The system should be able to perform a wide range of tasks without any user interaction at all." (Lukowicz et al. 2004, S. 232) Die Verfügung über einen Körper, der gewollte Bewegungen und Handlungen nicht blockiert, sondern störungsfrei 
umsetzt, gilt traditionell als eine wichtige Bedingung für Autonomie.

Mit der Verknüpfung von Datenerhebung, -verarbeitung und -versendung werden die Systeme aber auch offen für externe Einflüsse. Damit kommt ein in der Gegenwart zunehmend wichtigerer Aspekt der Autonomie ins Spiel: die informationelle Selbstbestimmung. Bei der Versendung von Körperdaten an externe Empfänger fordert die Autonomie, dass der Benutzer solcher Systeme selbst weiß und kontrollieren kann, welche Informationen über ihn versendet werden. Wahrscheinlich kann er nicht über jedes einzelne Datum informiert sein, aber zumindest über die Art und den Gegenstandsbereich der Information (Organe, Körperfunktionen, Lokalisation des Trägers etc.). Ferner müssen der externe Zugang und die Weitergabe der Daten geschützt bzw. autorisiert sein. Das ist auch ein Ziel der technologischen Entwicklung.

Aber gerade dieses Ziel kann mit zwei anderen angestrebten Zielen kollidieren: Zum einen mit dem Ziel der Zeitersparnis etwa in Notfällen. Welche Informationen oder Daten in solchen Fällen aufgenommen und weitergeleitet werden oder welche Reaktionen von den tragbaren oder implantierten Geräten ausgehen, kann wegen der Kürze der Zeit und der evtl. herabgesetzten Einwilligungsfähigkeit der Betroffenen u. U. nicht autorisiert werden. Zumindest die für die bisherigen Geräte geltenden datenschutzrechtlichen Regeln müssten daher unbedingt beibehalten werden. Zum anderen kann die informationelle Selbstbestimmung in Spannung geraten mit der angestrebten Überwachung oder den Hilfen für Beeinträchtigungen (etwa die Lokalisation von Verwirrten außerhalb sicherer Zonen). Derartige Hilfen betreffen etwa bei mentalen Beeinträchtigungen Akteure, die zu einer informierten und bewussten Selbstbestimmung gar nicht fähig sind. Bereits das Tragen der Geräte kann ihnen möglicherweise nicht hinreichend erklärt werden. Wichtige, sich daran anschließende Fragen lauten: $\mathrm{Zu}$ wie viel ,,mutmaßlicher Einwilligung" und wohlwollendem Paternalismus ist man in solchen Fällen berechtigt? Können die Träger nicht in solchen Fällen auch dem Diktat ihrer „Helfer“ ausgeliefert werden, etwa was Risiken oder Gewohnheiten der Lebensführung angeht? Wird ,pervasive“ Computing dann nicht zumindest zum ,persuasiven“, wenn nicht zur Steuerung von außen?
Probleme des Umganges mit Nicht-Einwilligungsfähigen, die in anderen Zusammenhängen (etwa der medizinischen Forschung) schon diskutiert worden sind, kehren hier unter einem neuen Aspekt wieder. Es muss nicht nur der objektive Vorteil, sondern auch die Vermutung der individuellen Zustimmung des Betroffenen mit dem Einsatz der Geräte und den Funktionen, die sie ausüben, gut gesichert sein.

Zwischen der Unterstützung von Autonomie und der technischen Rückkehr eines starken Paternalismus liegt hier offenbar ein schmaler Grat. Zumal Techniker sich oft von der Faszination der technischen Möglichkeiten verführen lassen und nicht berücksichtigen, dass Selbstbestimmung ein Stück weit auch im Umgang mit eigenen Unvollkommenheiten Grenzen der Information und der Optionen bestehen kann. Geräte, die nicht perfekt eingestellt, automatisiert und lernfähig (,,intelligent") sind, lassen sich manchmal dem Willen und den Gewohnheiten des Benutzers leichter unterwerfen als perfektionierte.

Erheblich in Richtung der Orwellschen Überwachungsutopien des "Großen Bruders“ gehen Szenarien der Beeinflussung des Lebenswandels durch externe Einflüsse von Versicherungen, Arbeitgebern oder gar staatlichen Verwaltungen (Bohn et al. 2004). „Those who exercise or eat nutritious meals could be rewarded for their healthy behavior with mobile micropayments that they could then use to pay wireless monthly charges (...) or pay medical expenses." (Varshney 2003, S. 140) Dies ist noch ein ernst gemeinter Vorschlag aus dem Repertoire der Entwickler. Von Missbrauch ist hier erst die Rede, wenn an weitere Möglichkeiten der Beeinflussung gedacht wird: „... pervasive healthcare information could be abused by corporations in deciding who should be promoted, by insurance companies in refusing coverage for people with poor health, and by spouses and their attorneys in divorce cases." (ebd.) „Klare Richtlinien” des Datenzuganges, der Verarbeitung und Weitergabe sind dagegen eine notwendige, aber vielleicht nicht hinreichende Vorkehrung. Bei der Entwicklung und Zulassung solcher Geräte sollten auch EthikKommissionen beteiligt sein. 


\section{Verbesserung, Chancengleichheit und Gerechtigkeit}

Hilfsmittel gegen Dysfunktionen und Beeinträchtigungen sind in aller Regel auch Mittel zur Leistungssteigerung, wenn sie von Gesunden benutzt werden. Auch bei den Anwendungen von Informations- und Kommunikationstechnologien im Gesundheitswesen sind solche Möglichkeiten des ,enhancements“ in Fülle vorstellbar. Sensoren, Rechner und Sender, die in die Kleidung eingewoben, in ein Körpernetzwerk (body LAN) integriert und an externe Systeme angeschlossen sind, können nicht nur körperliche Leistungsfähigkeit steigern, sondern auch über Umweltinformationen (etwa körpereigene „Autopiloten“) die Bewegungsfreiheit und Handlungseffizienz erheblich verbessern (Bohn et al. 2004). Damit ergeben sich nicht nur für die medizinische Anwendung, sondern auch für die mögliche Leistungssteigerung Fragen der Chancengleichheit und der Verteilungsgerechtigkeit.

Schon bei der medizinischen Kompensation körperlicher und schicksalhafter Benachteiligung (z. B. durch Unfall oder Erkrankung) ist es in Übereinstimmung mit der sozialstaatlichen, religiös oder philosophisch begründeten Tradition wichtig, für das Leben oder erhebliche Unterschiede der Lebensqualität wichtige Leistungen nicht gänzlich an die private Kaufkraft zu binden. Wenn es, mit John Rawls (1975) formuliert, zu unseren Fairnessvorstellungen gehört, bei Erhaltung der Chancengleichheit die Situation der Schlechtestgestellten (,worst off") zu verbessern, dann müssen Menschen unabhängig von ihren privaten Mitteln, abgestuft nach dem Grad ihrer Beeinträchtigungen und Risiken, in die Lage versetzt werden, kompensierende und präventive Geräte zu erwerben, die sie handhaben können. Von der Vermeidung übermäßigen Sicherheitsstrebens und eines starken Paternalismus war schon die Rede.

Noch schwieriger wird es sein, den Erwerb von „intelligenten Funktionskleidern“ nicht zu einer Verzerrung der Chancengleichheit werden zu lassen, ohne die Freiheit des privaten Kaufs und Konsums zu beseitigen - man kann sich ja auch heute schon Funktionskleidung und leistungsfähige Autos oder Handys kaufen. Zumindest für den Bereich von Beruf und öffentlichen Aufgaben sollte aber der Arbeitgeber verpflichtet sein, jeden Bewerber und Mitarbeiter mit
Kleidung und anderen Formen von „wearable systems“ zu versorgen, die ihn vor signifikanten Wettbewerbsnachteilen bewahren. Nicht nur im Bereich der genetischen Manipulationen oder der leistungssteigernden Pharmaka, sondern auch im Bereich der „computerunterstützten“" Kleidung, Accessoires, ja des ganzen Körpers stellt sich die Problematik des enhancements, der Chancengleichheit und der möglichen Belastung der Solidargemeinschaft mit Kompensationsleistungen. Die Prinzipien der sozialen Gerechtigkeit, wie sie im Sozialstaat oder in den Anforderungen an die Solidargemeinschaft im Gesundheitswesen ,institutionalisiert“ sind, gehen ja davon aus, dass den „Schwachen“, die nicht zum Ausgleich gesundheitlicher und anderer körperlicher Nachteile gegenüber dem „Normalstandard“ in der Lage sind, Kompensationsleistungen zustehen (Siep 2006).

\section{Schlussfolgerungen}

Die vorangehenden Überlegungen sollen kein Plädoyer für eine „Heuristik der Furcht“ (Hans Jonas) sein. Man muss nicht wegen der Missbrauchsmöglichkeiten raten, die Chancen auf erhebliche Erleichterung des Lebens gerade für schwer oder chronisch Kranke, behinderte und alte Menschen nicht wahrzunehmen. Auch Möglichkeiten der Kosteneinsparungen sind ein lohnendes Ziel. Technologische Entwicklungen in einer modernen Gesellschaft sind unumgänglich mit Folgeproblemen und Missbrauchsgefahren verbunden. Sie lassen sich auch nicht gänzlich durch vorherige Abschätzungen vermeiden. Aber unter dem Druck der notwendigen Kostensenkung und der Faszination technischer (und ökonomischer) Entwicklungsmöglichkeiten dürfen die Gefahren für die Güter der Sicherheit, der Autonomie und der Gerechtigkeit jedenfalls nicht aus den Augen verloren werden.

Daher ist es wichtig, die Entwicklung auf diesem Gebiet durch Folgenabschätzungen und ethische Überlegungen $\mathrm{zu}$ begleiten; dies darf aber nicht erst bei klinischen Studien geschehen, bei denen Ethik-Kommissionen ohnehin hinzugezogen werden. Da die Entwicklungen in besonderem Maße die informationelle Selbstbestimmung betreffen, müssen Datenschutzvorschriften nicht nur beachtet, sondern auch weiterentwickelt werden. Da nicht alle Folgen vor- 
weggenommen oder sozusagen ,im Labor getestet" werden können, müssen auch bereits eingesetzte Geräte zurückgenommen oder korrigiert werden können. Auch weniger manifeste Gefahren wie ein übersteigertes Sicherheitsstreben oder Formen des Paternalismus durch die beschützende(n) Technik(er) sollten nicht übersehen werden. Abzuwägen sind vor allem die Güter der Lebenserleichterung und der Unterstützung autonomer Lebensführung auf der einen Seite gegenüber dem Schutz der Privatsphäre und der Unabhängigkeit von Fremdsteuerung, aber auch der Chancengleichheit im Zugang zu den technischen Möglichkeiten auf der anderen. Kosteneinsparungen und Gewinnmöglichkeiten dürfen erst in der „zweiten Reihe“ stehen. Im weiteren Horizont stehen die Probleme der Optimierung des Menschen (enhancement) und der Auflösung der Grenzen zwischen Körper und Umwelt (Araya 1995).

\section{Anmerkung}

1) Siehe dazu Lukowicz, der auf "the possibility of detecting a wide range of potential hazards and emergencies" hinweist (Lukowicz 2004, S. 237).

\section{Literatur}

Araya, A.A., 1995: Questioning Ubiquitous Computing: In: Proceedings of the 1995 ACM $23^{\text {rd }}$ Annual Conference on Computer Science. ACM Press. http://doi.acm.org/10.1145/259526.259560 (download 5.5.08)

Bardram, J.E.; Baldus, H.; Favela, J., 2007: Pervasive Computing in Hospitals. In: Bardram, J.E.; Mihailidis, A.; Wan, D. (Hg.): Pervasive Computing in Healthcare. Boca Raton, FL, S. 49-77

Bohn, J.; Coroama, V.; Langheinrich, M., et al., 2004: Living in a world of smart everyday objects - Social, economical and ethical implications. Human and Ecological Risk Assessment 10/5 (2004), S. 763-785

Korhonen, I.; Bardram, J.E., 2004: Guest Editorial: Introduction to the Special Section on Pervasive Healthcare. In: IEEE Transactions on Information Technology in Biomedicine 8/3 (2004), S. 229-234.

Lukowicz, P.; Kirstein, T.; Tröster, G., 2004: Wearable Systems for Health Care Applications. In: Methods of Information Medicine 43 (2004), S. 232-238

Rawls, J., 1975: Eine Theorie der Gerechtigkeit. Frankfurt a. M.
Siep, L., 2006: Die biotechnische Neuerfindung des Menschen. In: Ach, S.; Pollmann, A. (Hg.): no body is perfect. Baumaßnahmen am menschlichen Körper - Bioethische und ästhetische Aufrisse. Bielefeld, S. 21-42

Siep, L., 2007: Ethik und Telemedizin. In: AnyCare (Hg.): Telemedizin - Innovationen für ein effizientes Gesundheitsmanagement. Stuttgart, S. 65-77

Varshney, U., 2003: Pervasive Medicine. In: IEEE Computer 36/12 (2003), S. 138-40

\section{Kontakt}

Prof. Dr. Ludwig Siep

Philosophisches Seminar

Westfälische Wilhelms-Universität

Domplatz 23, 48143 Münster

Tel.: +49 (0) 251 / 8324468

$《 》$ 